\title{
Pre-Mild Cognitive Impairment: Can Visual Memory Predict Who Rapidly Convert to Mild Cognitive Impairment?
}

\author{
Eun Hyun Seo ${ }^{1,2}$, Hoowon Kim ${ }^{1,3}$, Kyu Yeong Choi ${ }^{1,2}$, Kun Ho Lee ${ }^{1,4}$, and IL Han Choo ${ }^{1,5} \bowtie$ \\ ${ }^{1}$ National Research Center for Dementia, Gwangju, Republic of Korea \\ ${ }^{2}$ Premedical Science, College of Medicine, Chosun University, Gwangju, Republic of Korea \\ ${ }^{3}$ Department of Neurology, School of Medicine, Chosun University, Chosun University Hospital, Gwangju, Republic of Korea \\ ${ }^{4}$ College of Natural Sciences, Chosun University, Gwangju, Republic of Korea \\ ${ }^{5}$ Department of Neuropsychiatry, School of Medicine, Chosun University, Chosun University Hospital, Gwangju, Republic of Korea
}

\begin{abstract}
Objective Little is known about the natural course of pre-mild cognitive impairment (pre-MCI) and predictors to MCI. We followedup individuals with pre-MCI and cognitively normal (CN) elders to identify neuropsychological predictors for rapid conversion to MCI.

Methods Seventy-seven individuals with pre-MCI and $180 \mathrm{CN}$ elders were recruited from the pool of individuals registered at the National Research Center for Dementia in Gwangju, Korea. We followed-up with them after a mean of $14 \pm 2.29$ months. All participants underwent comprehensive clinical and neuropsychological assessment. Logistic regression analysis examined the ability of neuropsychological tests to predict conversions to MCI.

Results Of 257 participants, 142 (55.3\%) were eligible for the follow-up study (102 CN, 40 pre-MCI). Logistic regression revealed that spatial delayed recall significantly predicted the conversion from pre-MCI to MCI. In CN, copy for a complex figure significantly predicted the conversion to pre-MCI or MCI.

Conclusion Our findings indicated that spatial delayed recall was associated with rapid conversion from pre-MCI to MCI. Spatial organization and planning, measured by complex figure reproduction, were associated with rapid conversion from CN to pre-MCI or MCI. Our study suggests that inclusion of visuospatial reproduction and memory using a complex figure further facilitates early detection of MCI.

Psychiatry Investig 2018;15(9):869-875
\end{abstract}

Key Words Mild cognitive impairment, Pre-mild cognitive impairment, Early diagnosis, Alzheimer disease, Spatial memory.

\section{INTRODUCTION}

Over the last decade, the focus of the diagnostic framework for Alzheimer's disease (AD) has been gradually shifted toward very early stages, such as the preclinical asymptomatic state. ${ }^{1} \mathrm{~A}$ new disease model of $\mathrm{AD}$ stresses risk factor assessment, early detection, and intervention. ${ }^{2}$ In this context, mild cognitive impairment (MCI), which is known as a precursor of dementia including $\mathrm{AD},{ }^{3}$ has been a research focus. In particular, considerable number of recent studies provid-

Received: March 21, 2018 Revised: July 10, 2018

Accepted: July 29, 2018

$\triangle$ Correspondence: IL Han Choo, MD, PhD

Department of Neuropsychiatry, School of Medicine, Chosun University, Chosun University Hospital, 365 Pilmun-daero, Dong-gu, Gwangju 61452, Republic of Korea

Tel: +82-62-220-3104, Fax: +82-62-225-3659

E-mail: ilhan.choo@chosun.ac.kr

(a) This is an Open Access article distributed under the terms of the Creative Commons Attribution Non-Commercial License (https://creativecommons.org/licenses/bync/4.0) which permits unrestricted non-commercial use, distribution, and reproduction in any medium, provided the original work is properly cited. ed progression rates from $\mathrm{MCI}$ to $\mathrm{AD}$ dementia and aimed to improve the accuracy of predictors of such conversion. ${ }^{4-14}$ However, individuals with MCI already experience disturbed cognitive function, and although it is generally very mild, it can be also challenging for these individuals as well as their family. Therefore, reliable prediction markers for MCI would help to accelerate intervention.

Surprisingly fewer studies, however, have focused on predictors of MCI than on predictors of AD. Studies on MCI prediction have shown inconsistent results. For example, some of the studies reported that poor episodic memory performance was a risk factor for developing MCI, ${ }^{15,16}$ but one recent study reported that poor executive function, not episodic memory, at baseline was associated with a future MCI diagnosis. ${ }^{17}$ Additionally, there were other studies that demonstrated that poor everyday functioning at baseline was associated with a future MCI diagnosis, even after adjusting for cognitive abilities. ${ }^{18,19}$ Therefore, effective neuropsychological markers for predicting $\mathrm{MCI}$ remain unclear. 
Pre-mild cognitive impairment (pre-MCI) is an intermediate state between cognitively normal $(\mathrm{CN})$ and $\mathrm{MCI} .{ }^{3,20} \mathrm{In}$ dividuals with pre-MCI exhibit mild functional changes measured by Clinical Dementia Rating (CDR) scale, but their objective cognitive change is so subtle that it fall within normal range and does not meet MCI criteria. The label of "preMCI" is one of various terms indicating the status between normal aging and MCI, for example, subjective cognitive impairment (SCI), ${ }^{21}$ or subjective cognitive concerns. ${ }^{22}$ Among such terms pre-MCI could be the most later stage in the continuum between $\mathrm{CN}$ and MCI. However, whether pre-MCI can be considered as a precursor to MCI or not remained unclear. For early detection and effective treatment of $\mathrm{AD}$ and $\mathrm{MCI}$, a clinical course for pre-MCI would be of a large interest. In spite of the importance of pre-MCI in clinical and research settings, little is known about the natural course of pre$\mathrm{MCI}$ and predictors of the progression to subsequent decline, such as MCI or dementia.

Only two studies performed followed-up approach for preMCI. ${ }^{3,23}$ One longitudinal study reported higher rates of progression to $\mathrm{MCI}$ or $\mathrm{AD}$ than to $\mathrm{CN}$, suggesting that pre-MCI is a true precursor to MCI. ${ }^{23}$ However, these studies examined dementia predictors, not MCI predictors. It is necessary to investigate baseline neuropsychological characteristics of individuals with pre-MCI who will progress to MCI and those of individuals who will remain stable.

In the current study, therefore, we prospectively followed individuals with pre-MCI and CN elders for about one year. We investigated the conversion rate from pre-MCI to MCI and compared with that of $\mathrm{CN}$. We also aimed to identify clinical and neuropsychological prediction markers for rapid conversion to MCI.

\section{METHODS}

\section{Participants}

At baseline, 77 individuals with pre-MCI and $180 \mathrm{CN}$ elderly individuals were recruited from a pool of individuals registered at the National Research Center for Dementia in Gwangju, Korea, from January 2014 to July 2015. We followed-up with them after a mean of $14 \pm 2.29$ months. Detailed information regarding participants at baseline has been reported previously. ${ }^{20}$ Briefly, individuals with pre-MCI were diagnosed as such if their CDR score was 0.5 (memory subdomain is 0.5 ), and their neuropsychological performance was within a normal range, that is, all test $\mathrm{z}$-scores were above -1.5 according to age-, education-, and gender-specific norms. According to Morris et al, the global CDR score of 0.5 includes three different groups: 1) those who show impairment in memory and at least 3 of the 5 remaining CDR domains [CDR
0.5/Dementia of Alzheimer Type (DAT)], 2) those who show impairment in memory and 2 or fewer remaining CDR domains (CDR 0.5/incipient DAT), and 3) those who show impairment in memory only or the impairment is doubtful (CDR $0.5 /$ uncertain dementia). ${ }^{24}$ Our participants with pre-MCI were all in the category of the third one. All CN participants had a CDR score of 0 and neuropsychological performance within a normal range. Exclusion criteria for all participants were as follows: 1) evidence of focal brain lesions on MRI, including lacunes and white matter hyperintensity lesions of grade 2 or more, according to the Fazeka scale; ${ }^{25}$ 2) illiteracy; 3) severe visual or hearing loss; 4) any type of dementia or MCI; 5) any significant neurologic, medical, or psychiatric disorders that could affect mental function; and 6) current use of psychoactive medications.

\section{Baseline clinical and neuropsychological assessment}

All participants were examined with a clinical interview, which included an assessment of CDR. The CDR is a widely used measure of dementia severity that provides 5 levels of impairment (staging $0,0.5,1,2$, or 3 ) and has 6 subdomains (memory, orientation, judgment and problem solving, function in community affairs, home and hobbies, and personal care). ${ }^{26,27}$ The CDR sum of boxes (SOB) score is calculated with possible scores ranging from 0 to 18 . Medical history, including stroke or family history of dementia, and instrumental activities of daily living (IADL) ${ }^{28}$ were assessed. The Korean version of the Dementia Screening Questionnaire (DSQ), ${ }^{29}$ a self-report format of cognitive screening tool, was also administered. It consists of 15 items with a 3-point scale regarding general cognitive changes in everyday life over the past 1 year. The cutoff score was suggested to be $6 .{ }^{29}$ In addition, the Subjective Memory Complaints (SMC) ${ }^{30}$ and the Geriatric Depression Scale (GDS) ${ }^{31}$ were also administered in a self-report format.

A comprehensive neuropsychological assessment was performed using the Seoul Neuropsychological Screening Battery (SNSB), which covers five cognitive domains. ${ }^{32}$ The attention domain was assessed using a forward and backward digit span test. The language domain was assessed using a shortened version of the Boston Naming Test (BNT; 15 item version, Form A). The visuospatial domain was assessed using the copying test from the Rey Complex Figure Test (RCFT copy). The memory domain was assessed by six measures, including the Seoul Verbal Learning Test (SVLT) immediate recall (SVLT_imm), 20-minute delayed recall (SVLT_delayed), and yes-no recognition (SVLT_rec), RCFT immediate recall (RCFT_imm), 20-minute delayed recall (RCFT_delayed), and yes-no recognition (RCFT_rec). The frontal/executive domain was assessed using category fluency tests (animal and super- 
market lists), a phonemic fluency test (total score for ' $\neg$ ' /g/, '人' /s/, and 'O' /y/), the Stroop test (color naming in colorword incongruent condition), and Trail Making Tests (TMT) $\mathrm{A}$ and $\mathrm{B}$. The global cognition was assessed using the Mini Mental State Examination (MMSE).

\section{Follow-up assessment}

Each participant underwent the same clinical interview as they did in the baseline evaluation, including the CDR designation and neuropsychological assessment procedure. MCI was diagnosed according to consensus criteria: ${ }^{33} 1$ ) the person is neither normal nor demented; 2) there is evidence of cognitive deterioration shown by either decline measured objectively over time and/or a subjective report of decline by the self and/or an informant, in conjunction with objective cognitive deficits; and 3) activities of daily living are preserved, and complex instrumental functions are either intact or minimally impaired. In terms of the criteria for 2), objective cognitive change was identified by the neuropsychological performance $\mathrm{z}$-scores (1.5 and below) mentioned above. The final clinical diagnosis was made after reviewing all the available information in the consensus case conferences.

\section{Statistical analysis}

The pre-MCI group was divided into 2 subgroups according to diagnosis at the time of the second evaluation: those who converted to $\mathrm{MCI}$ (pre- $\mathrm{MCI}_{\mathrm{con}}$ ), and those who did not convert to $\mathrm{MCI}$ (pre- $\mathrm{MCI}_{\text {noncon }}$ ). All individuals who were stable or reverted to $\mathrm{CN}$ were included in the pre- $\mathrm{MCI}_{\text {noncon }}$ group. The $\mathrm{CN}$ group was also divided into 2 subgroups according to diagnosis at the time of the second evaluation: those who converted to $\mathrm{MCI}$ or pre-MCI $\left(\mathrm{CN}_{\mathrm{con}}\right)$, and those who were stable $\mathrm{CN}\left(\mathrm{CN}_{\text {noncon }}\right)$. Demographic and clinical data were compared between groups using separate oneway analyses of variance (ANOVA) and $\chi^{2}$ tests for continuous and categorical variables, respectively. For neuropsychological performance, $\mathrm{z}$-scores according to age-, education-, and gender-specific norms were compared between groups using oneway ANOVAs. Multivariable logistic regression analysis with stepwise selection was performed in order to examine the ability of neuropsychological tests to predict conversions. These analyses were performed using SPSS version 21.0 for Windows (IBM Corp., Armonk, NY, USA); p-values less than 0.05 were considered significant.

\section{Ethical approval}

The Institutional Review Board of Chosun University Hospital approved the present study (CHOSUN 2013-12-018053). Written informed consent was obtained from each participant.

\section{RESULTS}

\section{Participant characteristics}

Of 257 participants, 142 (55.3\%) were eligible for the follow-up study (102 CN, 40 pre-MCI). The reasons for dropout were: loss of contact $(n=19)$, refusal to participate $(n=63)$, poor medical condition $(n=19)$, and other $(n=14)$. The follow-up rate was $56.7 \%$ for $\mathrm{CN}$ and $51.9 \%$ for pre-MCI, and there were no group differences in the follow-up rates (Figure 1 ). When we compared baseline characteristics of subjects who completed follow-up (FU) and those who did not FU (no FU), men (65.8\%) followed up more than women (50.6\%). Digit span forward $(\mathrm{p}=0.039)$ and MMSE score $(\mathrm{p}=0.003)$ in FU were higher than in no FU. There were no differences between these two groups by age, education, GDS, SMC, ADL, DSQ, and other NP scores.

In the pre-MCI group, 13 (33\%) converted to MCI and were labeled pre-MCI $\mathrm{Mnn}_{\text {con }}$ In contrast, 18 (45\%) were stable pre-MCI and $9(23 \%)$ were reverted to $\mathrm{CN}$, and they were la-

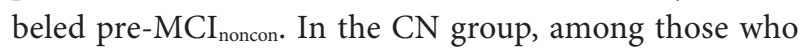
completed follow-up assessment, 7 (7\%) and 8 (8\%) converted to MCI and pre-MCI, respectively. These were labeled as $\mathrm{CN}_{\text {con }}$. In contrast, 73 (73\%) were stable $\mathrm{CN}$ and were labeled $\mathrm{CN}_{\text {noncon}}$. Significantly more individuals in the preMCI group converted to MCI than in $\mathrm{CN}$ group $\left(\chi_{\mathrm{df}=1}^{2}=15.61\right.$, $\mathrm{p}<0.001)$. Individuals $(\mathrm{n}=14,14 \%)$ who were stable with a CDR score of 0 but showed an MRI abnormality (evidence of focal brain lesions on MRI, including lacunes and white matter hyperintensity lesions of grade 2 or more) or a cognitive abnormality (test z-scores were below -1.5 according to age-, education-, and gender-specific norms) at the 2 nd evalua-

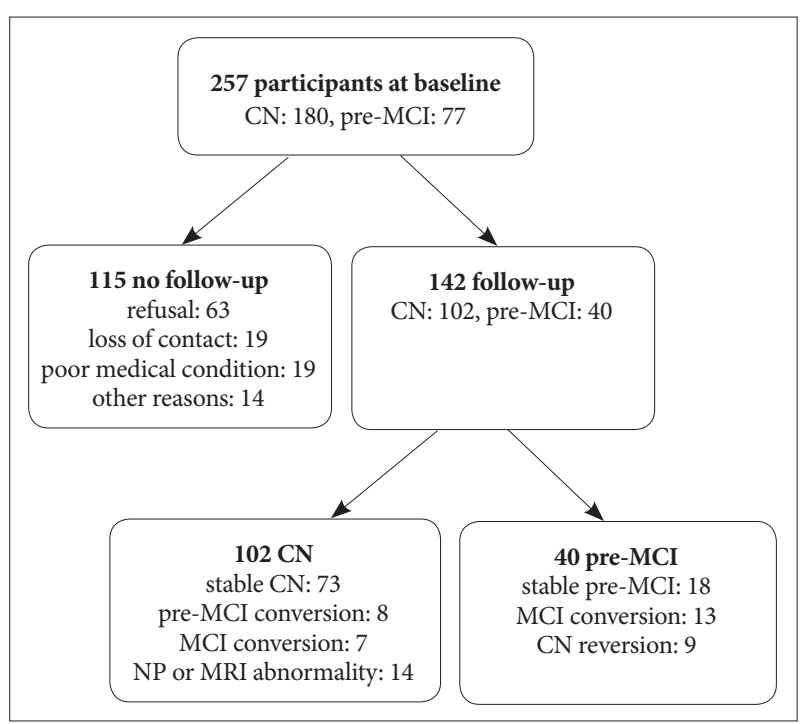

Figure 1. Flow chart of follow-up study. CN: cognitively normal, pre-MCl: pre-mild cognitive impairment, $\mathrm{MCl}$ : mild cognitive impairment. 
tion were excluded from the further analysis.

\section{Baseline characteristics of converters and non-converters}

In the pre-MCI group, the converters were older $(\mathrm{p}=0.048)$, and showed lower SVLT_rec score $(\mathrm{p}=0.018)$, and lower RCFT _delayed score $(\mathrm{p}=0.042)$ (Table 1) than those in pre- $\mathrm{MCI}_{\text {noncon. }}$. In the $\mathrm{CN}$ group, the converters' education levels were lower $(\mathrm{p}=0.018)$ and DSQ scores were higher $(\mathrm{p}=0.013)$ than those in $\mathrm{CN}_{\text {noncon. }}$. The converters also showed lower BNT scores ( $\mathrm{p}=$ $0.035)$, RCFT copy scores $(\mathrm{p}=0.001)$, and marginally lower RCFT_rec scores ( $\mathrm{p}=0.059$ ) (Table 2). In both groups, there were no significant differences between converters and non-converters in SMC, GDS, IADL, and rest of the neuropsychological tests, including MMSE.

\section{Prediction models for the conversion to MCI}

Given the significantly different scores that resulted from the ANOVA tests, multivariable logistic regression analysis with stepwise selection was performed to examine the ability of such scores to predict conversions. In the pre-MCI group, only the RCFT_delayed score significantly predicted the conversion to $\mathrm{MCI}(\mathrm{OR}=0.26 ; 95 \% \mathrm{CI}, 0.07-0.96 ; \mathrm{p}=0.044)$ (Table 3). In the CN group, only the RCFT copy score (OR $=0.15$; $95 \% \mathrm{CI}, 0.04-0.61 ; \mathrm{p}=0.008)$ significantly predicted the conversion to pre-MCI or MCI.

\section{DISCUSSION}

The identification of individuals in very early stages of the $\mathrm{AD}$ spectrum is a high priority in both research and clinical settings. We prospectively followed pre-MCI and $\mathrm{CN}$ older adults who were all recruited from the community. We compared baseline characteristics of neuropsychological measures between converters and non-converters at a one-year followup. Our study revealed that although objective neuropsychological performance is within normal, individuals with preMCI were more likely to convert to MCI than CN elderly individuals. It also suggested that RCFT copy and delayed recall performance acted as prognostic markers for rapid conversion to $\mathrm{MCI}$ or pre-MCI.

Among individuals with pre-MCI, 33\% were converted to $\mathrm{MCI}$ at a one-year follow-up. Among $\mathrm{CN}$ individuals, $7 \%$ were converted to MCI at a one-year follow-up, which is largely consistent with the findings of previous studies that used community-based recruitment. ${ }^{15-17,22}$ Eight percent of $\mathrm{CN}$ were converted to pre-MCI, which is also similar to the findings in previous reports. ${ }^{23}$ Compared to the $\mathrm{CN}$ conversion rate, individuals with pre-MCI were significantly more liked to convert. Very few studies have focused on the clinical course of
Table 1. Clinical and neuropsychological characteristics at baseline in pre-mild cognitive impairment subgroups*

\begin{tabular}{|c|c|c|c|}
\hline & preMCI $_{\text {con }}$ & preMCI $_{\text {noncon }}$ & p-value \\
\hline $\mathrm{N}$ & 13 & 27 & \\
\hline Age (SD), $\mathrm{y}^{\dagger}$ & $75.54(4.63)$ & $72.67(3.93)$ & 0.048 \\
\hline Education (SD), y & $12.08(4.65)$ & $10.30(4.30)$ & 0.240 \\
\hline Female, N & 6 & 21 & 0.072 \\
\hline SMC & $3.31(2.78)$ & $4.63(3.38)$ & 0.229 \\
\hline IADL & $0.12(0.14)$ & $0.10(0.13)$ & 0.547 \\
\hline GDS & $6.54(4.65)$ & $8.19(5.40)$ & 0.352 \\
\hline MMSE & $-0.18(0.75)$ & $0.04(0.71)$ & 0.376 \\
\hline \multicolumn{4}{|l|}{ Attention } \\
\hline DSF & $-0.44(1.27)$ & $0.62(1.00)$ & 0.627 \\
\hline DSB & $0.20(1.55)$ & $0.37(0.93)$ & 0.661 \\
\hline \multicolumn{4}{|l|}{ Language } \\
\hline BNT & $0.37(0.82)$ & $0.30(0.93)$ & 0.811 \\
\hline \multicolumn{4}{|c|}{ Visuospatial functions } \\
\hline RCFT copy & $0.31(0.60)$ & $0.51(0.44)$ & 0.256 \\
\hline \multicolumn{4}{|l|}{ Memory } \\
\hline SVLT_imm & $-0.18(0.38)$ & $0.15(1.03)$ & 0.267 \\
\hline SVLT_delayed & $-0.29(0.67)$ & $0.22(1.06)$ & 0.124 \\
\hline SVLT_rec $^{\dagger}$ & $-0.30(0.91)$ & $0.39(0.79)$ & 0.018 \\
\hline RCFT_imm & $-0.05(1.03)$ & $0.24(0.91)$ & 0.357 \\
\hline RCFT_delayed $^{\dagger}$ & $-0.12(0.64)$ & $0.37(0.73)$ & 0.042 \\
\hline RCFT_rec & $0.01(0.81)$ & $0.34(0.76)$ & 0.202 \\
\hline \multicolumn{4}{|l|}{ Executive functions } \\
\hline Fluency_A & $-0.03(0.83)$ & $0.04(0.75)$ & 0.783 \\
\hline Fluency_P & $-0.32(0.97)$ & $0.27(0.83)$ & 0.055 \\
\hline Stroop & $0.04(0.89)$ & $0.40(0.91)$ & 0.250 \\
\hline TMT_A & $-0.02(0.53)$ & $0.29(0.52)$ & 0.089 \\
\hline TMT_B & $0.36(0.67)$ & $0.24(0.60)$ & 0.585 \\
\hline
\end{tabular}

*neuropsychological data presented as group mean $\mathrm{z}$-scores based on age-, education-, and gender specific normative information (standard deviation), ${ }^{\dagger}$ significant group-difference. pre- $\mathrm{MCI}_{\mathrm{con}}$ : pre-mild cognitive impairment group who converted to mild cognitive impairment, pre- $\mathrm{MCI}_{\text {noncon }}$ : pre-mild cognitive impairment group who did not convert to MCI, SMC: subjective memory complaints, IADL: instrumental activities of daily living, GDS: geriatric depression scale, DSQ: dementia screening questionnaire, MMSE: mini mental status examination, DSF: digit span forward, DSB: digit span backward, BNT: Boston naming test (15 item), RCFT copy: Rey complex figure test copy score, SVLT_imm: Seoul verbal learning test, immediate recall score, SVLT_delayed: SVLT, delayed recall score, SVLT_rec: SVLT, recognition score, RCFT_ imm: RCFT, immediate recall score, RCFT_delayed: RCFT, delayed recall score, RCFT_rec: RCFT, recognition score, Fluency_A: fluency score for animal, Fluency_P: fluency score for 3 Korean alphabet, Stoop: Stroop score for color naming in color-word in incongruent condition, TMT: trail making test 
Table 2. Clinical and neuropsychological characteristics at baseline in cognitively normal subgroups*

\begin{tabular}{|c|c|c|c|}
\hline & $\mathrm{CN}_{\text {con }}$ & $\mathrm{CN}_{\text {noncon }}$ & p-value \\
\hline $\mathrm{N}$ & 15 & 73 & \\
\hline Age (SD), y & $72.87(3.80)$ & $71.86(3.72)$ & 0.897 \\
\hline Education (SD), $\mathrm{y}^{\dagger}$ & $9.53(5.03)$ & $9.71(4.39)$ & 0.018 \\
\hline Female, $\mathrm{N}$ & 5 & 26 & 0.866 \\
\hline SMC & $3.27(3.29)$ & $2.82(2.44)$ & 0.547 \\
\hline IADL & $0.03(0.73)$ & $0.06(0.12)$ & 0.397 \\
\hline GDS & $7.87(3.70)$ & $7.27(5.21)$ & 0.677 \\
\hline $\mathrm{DSQ}^{\dagger}$ & $3.80(1.6)$ & $2.74(1.45)$ & 0.013 \\
\hline MMSE & $0.13(0.65)$ & $0.35(0.72)$ & 0.272 \\
\hline \multicolumn{4}{|l|}{ Attention } \\
\hline DSF & $0.36(1.23)$ & $0.37(1.15)$ & 0.993 \\
\hline DSB & $0.21(1.04)$ & $0.08(1.12)$ & 0.682 \\
\hline \multicolumn{4}{|l|}{ Language } \\
\hline $\mathrm{BNT}^{+}$ & $0.11(0.75)$ & $0.60(0.81)$ & 0.035 \\
\hline \multicolumn{4}{|c|}{ Visuospatial functions } \\
\hline RCFT copy ${ }^{\dagger}$ & $-0.01(0.86)$ & $0.57(0.54)$ & 0.001 \\
\hline \multicolumn{4}{|l|}{ Memory } \\
\hline SVLT_imm & $0.28(0.64)$ & $0.37(0.85)$ & 0.675 \\
\hline SVLT_delayed & $0.07(0.69)$ & $0.42(0.96)$ & 0.181 \\
\hline SVLT_rec & $0.33(0.61)$ & $0.56(0.64)$ & 0.204 \\
\hline RCFT_imm & $0.11(1.09)$ & $0.38(0.93)$ & 0.324 \\
\hline RCFT_delayed & $0.12(0.93)$ & $0.38(0.92)$ & 0.322 \\
\hline RCFT_rec & $0.59(0.45)$ & $0.20(0.76)$ & 0.059 \\
\hline \multicolumn{4}{|l|}{ Executive functions } \\
\hline Fluency_A & $0.31(0.88)$ & $0.57(0.96)$ & 0.337 \\
\hline Fluency_P & $0.23(0.85)$ & $0.33(0.96)$ & 0.726 \\
\hline Stroop & $0.68(0.65)$ & $0.63(0.74)$ & 0.838 \\
\hline TMT_A & $0.47(0.48)$ & $0.37(0.55)$ & 0.553 \\
\hline TMT_B & $0.33(0.63)$ & $0.44(0.62)$ & 0.556 \\
\hline
\end{tabular}

*neuropsychological data presented as group mean z-scores based on age-, education-, and gender specific normative information (standard deviation), ${ }^{\dagger}$ significant group-difference. $\mathrm{CN}_{\text {con }}$ : cognitively normal elders who converted to mild cognitive impairment or pre-mild cognitive impairment, $\mathrm{CN}_{\text {noncon: }}$ cognitively normal elders who were stable, SMC: subjective memory complaints, IADL: instrumental activities of daily living, GDS: geriatric depression scale, DSQ: dementia screening questionnaire, MMSE: mini mental status examination, DSF: digit span forward, DSB: digit span backward, BNT: Boston naming test (15 item), RCFT copy: Rey complex figure test copy score, SVLT_imm: Seoul verbal learning test, immediate recall score, SVLT_delayed: SVLT, delayed recall score, SVLT_rec: SVLT, recognition score, RCFT_imm: RCFT, immediate recall score, RCFT_delayed: RCFT, delayed recall score, RCFT_rec: RCFT, recognition score, Fluency_A: fluency score for animal, Fluency_P: fluency score for 3 Korean alphabet, Stoop: Stroop score for color naming in color-word in incongruent condition, TMT: trail making test
pre-MCI. Only two studies have explored the clinical course of pre-MCI longitudinally.,23 Our results largely correspond with these previous studies, for example, similar conversion rates (e.g., Duara et al. ${ }^{23}$ reported $28.6 \%$ progressed to $\mathrm{MCI}$ ) and significantly more converted to MCI than $\mathrm{CN}$. In terms of objective neuropsychological tests, individuals with $\mathrm{CN}$ and pre-MCI were all within a normal range of performance, but their CDR scores were 0 and 0.5 , respectively. As previous studies pointed out, $\mathrm{CDR}$ can detect intraindividual cognitive changes, ${ }^{3,23}$ and therefore, it may improve sensitivity for detecting very early cognitive changes, even when objective cognitive function is within a normal limit., ${ }^{3,2}$ Along with previous evidence, the present results also support that identification of pre-MCI using the CDR system is helpful for the early detection of cognitive changes in a longitudinal perspective.

Our findings that pre-MCI converters were older and showed lower levels of verbal and spatial memory performance than non-converters at baseline suggest age and memory measure are sensitive to early changes in the $\mathrm{AD}$ spectrum. This is not surprising, because old age and poor memory function are well-known risk factors for dementia in both $\mathrm{CN}$ and MCI individuals. Further logistic regression analysis indicated that pre-MCI elderly individuals with higher performances of spatial memory (i.e., RCFT_delayed score) had decreased probability of conversion to MCI from pre-MCI. Specifically, preMCI elderly individuals with higher RCFT_delayed scores had a 3.8 times higher probability to be stable pre-MCI than preMCI elderly individuals with lower RCFT_delayed scores. RCFT is a very complex figure and consists of 18 units. We speculate that RCFT delayed recall with an interval of 20 minutes would be much more difficult than word list delayed recall. Because the pre-MCI stage falls within a relatively earlier phase in the $\mathrm{AD}$ spectrum, memory measure with higher difficulty level might provide a more efficient assessment of whether pre-MCI individuals would progress to $\mathrm{MCI}$ or remain stable. However, there are few studies reporting spatial memory performance as a risk factor. One possible reason might be that a spatial memory measure was not included in many of previous studies. For example, several recent conversion studies ${ }^{8,12,13}$ had adopted only verbal memory measures.

On the other hand, $\mathrm{CN}$ converters showed lower levels of education, lower abilities of naming and RCFT copy, and more functional changes in everyday life than the non-converters. Among these variables, our logistic regression analysis indicated that CN elderly individuals with higher RCFT copy scores had decreased probability of conversion to pre-MCI or MCI from CN. Specifically, CN elderly individuals with higher RCFT copy scores had a 6.7 times higher probability to be stable $\mathrm{CN}$ than $\mathrm{CN}$ elderly individuals with lower RCFT copy scores. RCFT copy simply consists of reproducing a com- 
Table 3. Logistic regression model for conversion prediction

\begin{tabular}{|c|c|c|c|c|c|}
\hline Variables & Regression coefficient & Standard error & Odd ratio & $95 \% \mathrm{CI}$ & p-value \\
\hline \multicolumn{6}{|l|}{ Pre-MCI } \\
\hline Intercept & -15.98 & 7.96 & 0.00 & & \\
\hline Age & 0.21 & 0.11 & 1.23 & $0.10-1.52$ & 0.051 \\
\hline SVLT_rec & -0.93 & 0.50 & 0.39 & $0.15-1.05$ & 0.062 \\
\hline RCFT_delayed* & -1.33 & 0.66 & 0.26 & $0.07-0.96$ & 0.044 \\
\hline \multicolumn{6}{|l|}{$\mathrm{CN}$} \\
\hline Intercept & -2.70 & 1.82 & 0.07 & & \\
\hline Education & -0.06 & 0.14 & 0.94 & $0.72-1.23$ & 0.673 \\
\hline RCFT copy* & -1.93 & 0.73 & 0.15 & $0.04-0.61$ & 0.008 \\
\hline RCFT_rec & 2.09 & 1.15 & 8.08 & $0.84-77.53$ & 0.070 \\
\hline BNT & -2.02 & 1.13 & 0.13 & $0.01-1.23$ & 0.076 \\
\hline DSQ & 0.11 & 0.20 & 1.12 & $0.76-1.65$ & 0.578 \\
\hline
\end{tabular}

*significant association. CN: cognitively normal, pre-MCI: pre-mild cognitive impairment, DSQ: dementia screening questionnaire, BNT: Boston naming test (15 item), RCFT copy: Rey complex figure test copy score, SVLT_rec: SVLT, recognition score, RCFT_delayed: RCFT, delayed recall score, RCFT_rec: RCFT, recognition score

plex figure. It is a widely used neuropsychological tool for the assessment of visuospatial constructional ability. However, because of the high complexity of the task figure, it also involves cognitive processes regarding strategic planning and organization of a fractional figure into a meaningful perceptual one at the time of drawing the complex figure. ${ }^{34}$ Thus, RCFT has the potential to be a useful method for the assessment of frontal lobe or executive function, as well as visuospatial ability. In fact, some previous studies have reported that RCFT score was correlated with conventional executive function measures and that the score could differentiate subjects with either no, mild, or severe executive dysfunction. ${ }^{35,36}$ Therefore, the association between RCFT copy score and clinical progression in our study may reflect that alteration in executive function (i.e., organization and planning) can be a very early marker of MCI. This interpretation of the present study corresponds with the results of previous studies, which reported that poor executive function was associated with developing MCI or functional decline. ${ }^{17,37}$ In addition, a very early cognitive sign in the course of $\mathrm{AD}$ spectrum might be a poor executive function..$^{20}$

Some limitations and future directions should be discussed. First, the dropout rate was considerable during the follow-up process, and, consequently, the final sample size was small. Participants in the current study were all recruited from community. This study included individuals with $\mathrm{CN}$ and preMCI who had essentially normal performances on objective neuropsychological tests. Therefore, there were some individuals who had no need for further visits for clinical and cognitive evaluation. Future studies based on larger sample size, especially for pre-MCI group, are needed. Second, the present study has a relatively short follow-up period. Therefore, our results should be interpreted with caution. Predictors discussed in our study were limited to prediction of "rapid" conversion. A longer follow-up period would provide broader perspective. Third, our results were based on clinical and neuropsychological factors only. Inclusion of biological markers such as the apolipoprotein E (APOE) genotype or conduction of a neuroimaging study may increase predictive power in addition to allowing analysis of neuropsychological markers. Although biomarkers have the potential to show good prediction information, biomarker studies are not always feasible in clinical situations. Our study's focus on neuropsychological tests allows it to be more easily applicable to general clinical settings. Lastly, future conversion studies are advised to include sensitive measures of visuospatial reproduction and memory for complex figures in order to detect very early change in the $\mathrm{AD}$ spectrum.

In conclusion, our findings indicated that individuals with pre-MCI are more likely to convert to MCI compared to CN individuals. Performance of spatial delayed recall at baseline was especially associated with rapid conversion from preMCI to MCI. Moreover, spatial organization and planning, measured by reproduction of a complex figure, were associated with rapid conversion from $\mathrm{CN}$ to pre-MCI or MCI. Our study suggests that inclusion of measures of visuospatial reproduction and memory using a complex figure further facilitates early detection of MCI.

\section{Acknowledgments}

This research was supported by a Basic Science Research Program through the National Research Foundation of Korea (NRF), funded by the Ministry of Education (NRF-2017R1D1A1B03028377), and it was sup- 
ported by the Brain Research Program through the NRF funded by the Ministry of Science, ICT \& Future Planning (NRF-2014M3C7A1046041).

\section{REFERENCES}

1. Sperling RA, Aisen PS, Beckett LA, Bennett DA, Craft S, Fagan AM, et al. Toward defining the preclinical stages of Alzheimer's disease: recommendations from the National Institute on Aging-Alzheimer's Association workgroups on diagnostic guidelines for Alzheimer's disease. Alzheimers Dement 2011;7:280-292.

2. Dubois B, Hampel H, Feldman HH, Scheltens P, Aisen P, Andrieu S, et al. Preclinical Alzheimer's disease: Definition, natural history, and diagnostic criteria. Alzheimers Dement 2016;12:292-323.

3. Storandt M, Grant EA, Miller JP, Morris JC. Longitudinal course and neuropathologic outcomes in original vs revised MCI and in pre-MCI. Neurology 2006;67:467-473.

4. Clark DG, McLaughlin PM, Woo E, Hwang K, Hurtz S, Ramirez L, et al. Novel verbal fluency scores and structural brain imaging for prediction of cognitive outcome in mild cognitive impairment. Alzheimers Dement (Amst) 2016;2:113-122.

5. Ding D, Zhao Q, Guo Q, Liang X, Luo J, Yu L, et al. Progression and predictors of mild cognitive impairment in Chinese elderly: a prospective follow-up in the Shanghai Aging Study. Alzheimers Dement (Amst) 2016;4:28-36.

6. Mazzeo S, Santangelo R, Bernasconi MP, Cecchetti G, Fiorino A, Pinto $\mathrm{P}$, et al. Combining cerebrospinal fluid biomarkers and neuropsychological assessment: a simple and cost-effective algorithm to predict the progression from mild cognitive impairment to alzheimer's disease dementia. J Alzheimers Dis 2016;54:1495-1508.

7. Falahati F, Ferreira D, Muehlboeck JS, Eriksdotter M, Simmons A, Wahlund LO, et al. Monitoring disease progression in mild cognitive impairment: associations between atrophy patterns, cognition, APOE and amyloid. Neuroimage Clin 2017;16:418-428.

8. Gallucci M, Di Battista ME, Battistella G, Falcone C, Bisiacchi PS, Di Giorgi E. Neuropsychological tools to predict conversion from amnestic mild cognitive impairment to dementia. The TREDEM Registry. Neuropsychol Dev Cogn B Aging Neuropsychol Cogn 2018;25:550-560.

9. Gothlin M, Eckerstrom M, Rolstad S, Wallin A, Nordlund A. Prognostic accuracy of mild cognitive impairment subtypes at different cut-off levels. Dement Geriatr Cogn Disord 2017;43:330-341.

10. Kim JW, Byun MS, Sohn BK, Yi D, Seo EH, Choe YM, et al. Clinical dementia rating orientation score as an excellent predictor of the progression to Alzheimer's disease in mild cognitive impairment. Psychiatry Investig 2017;14:420-426.

11. Li K, Chan W, Doody RS, Quinn J, Luo S; Alzheimer's Disease Neuroimaging Initative. Prediction of conversion to Alzheimer's disease with longitudinal measures and time-to-event data. J Alzheimers Dis 2017; 58:361-371.

12. Liu K, Chen K, Yao L, Guo X. Prediction of mild cognitive impairment conversion using a combination of independent component analysis and the cox model. Front Hum Neurosci 2017;11:33.

13. Michaud TL, Su D, Siahpush M, Murman DL. The risk of incident mild cognitive impairment and progression to dementia considering mild cognitive impairment subtypes. Dement Geriatr Cogn Dis Extra 2017;7:15-29.

14. Pagani M, Nobili F, Morbelli S, Arnaldi D, Giuliani A, Oberg J, et al. Early identification of MCI converting to AD: a FDG PET study. Eur J Nucl Med Mol Imaging 2017;44:2042-2052.

15. Blacker D, Lee H, Muzikansky A, Martin EC, Tanzi R, McArdle JJ, et al. Neuropsychological measures in normal individuals that predict subsequent cognitive decline. Arch Neurol 2007;64:862-871.

16. Oulhaj A, Wilcock GK, Smith AD, de Jager CA. Predicting the time of conversion to $\mathrm{MCI}$ in the elderly: role of verbal expression and learning. Neurology 2009;73:1436-1442.

17. Chen Y, Denny KG, Harvey D, Farias ST, Mungas D, DeCarli C, et al.
Progression from normal cognition to mild cognitive impairment in a diverse clinic-based and community-based elderly cohort. Alzheimers Dement 2017;13:399-405.

18. Nowrangi MA, Rosenberg PB, Leoutsakos JS. Subtle changes in daily functioning predict conversion from normal to mild cognitive impairment or dementia: an analysis of the NACC database. Int Psychogeriatr 2016;28:2009-2018.

19. Farias ST, Lau K, Harvey D, Denny KG, Barba C, Mefford AN. Early functional limitations in cognitively normal older adults predict diagnostic conversion to mild cognitive impairment. J Am Geriatr Soc 2017;65:1152-1158.

20. Seo EH, Kim H, Lee KH, Choo IH. Altered executive function in premild cognitive impairment. J Alzheimers Dis 2016;54:933-940.

21. Reisberg B, Prichep L, Mosconi L, John ER, Glodzik-Sobanska L, Boksay I, et al. The pre-mild cognitive impairment, subjective cognitive impairment stage of Alzheimer's disease. Alzheimers Dement 2008; 4(1 Suppl 1):S98-S108.

22. Donovan NJ, Amariglio RE, Zoller AS, Rudel RK, Gomez-Isla T, Blacker $\mathrm{D}$, et al. Subjective cognitive concerns and neuropsychiatric predictors of progression to the early clinical stages of Alzheimer disease. Am J Geriatr Psychiatry 2014;22:1642-1651.

23. Duara R, Loewenstein DA, Greig MT, Potter E, Barker W, Raj A, et al. Pre-MCI and MCI: neuropsychological, clinical, and imaging features and progression rates. Am J Geriatr Psychiatry 2011;19:951-960.

24. Morris JC, Storandt M, Miller JP, McKeel DW, Price JL, Rubin EH, et al. Mild cognitive impairment represents early-stage Alzheimer disease. Arch Neurol 2001;58:397-405.

25. Fazekas F, Chawluk JB, Alavi A, Hurtig HI, Zimmerman RA. MR signal abnormalities at $1.5 \mathrm{~T}$ in Alzheimer's dementia and normal aging. AJR Am J Roentgenol 1987;149:351-356.

26. Hughes CP, Berg L, Danziger WL, Coben LA, Martin RL. A new clinical scale for the staging of dementia. Br J Psychiatry 1982;140:566-572.

27. Morris JC. The Clinical Dementia Rating (CDR): current version and scoring rules. Neurology 1993;43:2412-2414.

28. Kang SJ, Choi SH, Lee BH, Kwon JC, Na DL, Han SH, et al. The Reliability and Validity of the Korean Instrumental Activities of Daily Living (K-IADL). J Korean Neurol Assoc 2002;20:8-14.

29. Yang DW, Cho BL, Chey JY, Kim SY, Kim BS. The Development and Validation of Korean Dementia Screening Questionnaire (KDSQ). J Korean Neurol Assoc 2002;20:135-141.

30. Youn JC, Kim KW, Lee DY, Jhoo JH, Lee SB, Park JH, et al. Development of the subjective memory complaints questionnaire. Dement Geriatr Cogn Disord 2009;27:310-317.

31. Kim JY, Park JH, Lee JJ, Huh Y, Lee SB, Han SK, et al. Standardization of the korean version of the geriatric depression scale: reliability, validity, and factor structure. Psychiatry Investig 2008;5:232-238.

32. Kang Y, Na D, Hahn S. Seoul Neuropsychological Screening Battery. Incheon: Human Brain Research \& Consulting Co; 2003.

33. Winblad B, Palmer K, Kivipelto M, Jelic V, Fratiglioni L, Wahlund LO, et al. Mild cognitive impairment--beyond controversies, towards a consensus: report of the International Working Group on Mild Cognitive Impairment. J Intern Med 2004;256:240-246.

34. Watanabe K, Ogino T, Nakano K, Hattori J, Kado Y, Sanada S, et al. The Rey-Osterrieth Complex Figure as a measure of executive function in childhood. Brain Dev 2005;27:564-569.

35. Somerville J, Tremont G, Stern RA. The Boston Qualitative Scoring System as a measure of executive functioning in Rey-Osterrieth Complex Figure performance. J Clin Exp Neuropsychol 2000;22:613-621.

36. Ogino T, Watanabe K, Nakano K, Kado Y, Morooka T, Takeuchi A, et al. Predicting executive function task scores with the Rey-Osterrieth Complex Figure. Brain Dev 2009;31:52-57.

37. Johnson JK, Lui LY, Yaffe K. Executive function, more than global cognition, predicts functional decline and mortality in elderly women. J Gerontol A Biol Sci Med Sci 2007;62:1134-1141. 